\title{
Modifiable and Non-Modifiable Risk Factors of Stroke: A Review Update
}

\author{
MS Jahirul Hoque Choudhury ${ }^{1}$, Md Tauhidul Islam Chowdhury², Abu Nayeem, \\ Waseka Akter Jahan ${ }^{4}$ \\ ${ }^{1}$ Assistant Professor, Dept. of Clinical Neurology, National Institute of Neurosciences \& Hospital, \\ Dhaka, Bangladesh; ${ }^{2}$ Assistant Professor, Dept. of Clinical Neurology, National Institute \\ of Neurosciences \& Hospital, Dhaka, Bangladesh; ${ }^{3}$ Assistant Professor, Department \\ of Neuromedicine, National Institute of Neurosciences \& Hospital, Dhaka, \\ Bangladesh; ${ }^{4}$ Assistant Professor, Department of Biochemistry, \\ National Institute of Neurosciences \& Hospital, \\ Dhaka, Bangladesh;
}

Received on: 24 July 2014; Reviewed on: 26 September 2014; Published on: 1 January 2015

\begin{abstract}
Stroke is the third most common cause of death in industrialized countries. Stroke is the most important cause of morbidity and longterm disability in Europe as well as in other industrialized nations. Prevalence rate was higher among men compared with women 3.44 and 2.41 per 1000 respectively. Data from the Northern Manhattan study showed the age adjusted incidence of first ischemic stroke per 100,000 was 88 in Whites 191 in Blacks and 149 in Hispanics. Black has almost thrice the risk of first ever stroke compared with Whites. The age adjustment stroke incidence rates for first ever stroke are 167 for White males, 138 for White females, 323 for Black males and 260 for Black females. Among American-Indian age 65-74, the annual rates per 1,000 population of new and recurrent stoke are 6.1 for men and 6.6 for women. Stroke accounted for about one of every 15 deaths in the United State in 2003. About 50 percent of these deaths occurred out of hospital. On average, about every three minutes someone dies of a stroke. In this review the modifiable and non-modifiable risks factors are discussed. [J Natl Inst Neurosci Bangladesh 2015;1(1):22-26]

Keywords: Stroke, risk factors, modifible

Correspondence : MS Jahirul Hoque Choudhury, Assistant Professor, Department of Clinical Neurology, National Institute of Neurosciences \& Hospital, Dhaka-1207, Bangladesh; Email: dr_jahirulhc@yahoo.com; Cell No.:+8801819122458

Conflict of Interest: None

Contribution to Authors: All authors contribute during the preparation of article.

How to cite the articale: Choudhury MSJH, Choudhury MTI, Nayeem A, Jahan WA. Modifiable and Non-Modifiable Risk Factors of Stroke: A Review Update. J Natl Inst Neurosci Bangladesh 2015;1(1): 22-26
\end{abstract}

\section{Introduction}

WHO defines stroke as a clinical syndrome characterized by rapidly developing clinical symptoms and/or signs of focal and at times global applied to patients in deep coma and those with subarachnoid hemorrhage, loss of cerebral function, with symptoms lasting for more than 24 hours or leading to death, with no apparent cause other than that of vascular origin ${ }^{1}$. The incidence of stroke varies in different European countries. It is estimated to be between 100 and 200 new strokes / 100,000 inhabitants / year. This imposes an enormous economic burden ${ }^{2}$. The best UK data, from the Oxford shire Community stroke project shows incidence rate for first stroke is 1.6 per 1000 person years at risk $^{3}$. Risk increased rapidly with age. In many recent series, mortality is about $15.0 \%$ at one month, $30.0 \%$ at one year and $50.0 \%$ at five years ${ }^{4}$. From the early 1970 s to early 1990 s the estimated number of non-institutionalized stroke survivors increased from 1.5 million to 2.4 million $^{5}$.
The prevalence of stroke in American Indian mean age 4574 ranges from 0.2 to 1.4 percent and in women from 0.2 to 0.7 percent $^{5}$. In a study in Bangladesh the prevalence rate of stroke were $2.0,3.0,2.0,10.0$, and 10.0 per 1000 within age groups of 40 -49 years, 50-59 years, 60-69 years, 70-79 years and 80 years to above age group respectively ${ }^{6}$. Prevalence rate rose with age. People with age range 70-79 years compared to $40-49$ years age range is 4.98 times and people with age range $>80$ years compared to $40-49$ years age range is 4.78 times more likely to have suffered from stroke. Prevalence rate was higher among men compared with women 3.44 and 2.41 per 1000 respectively ${ }^{7}$. On average every 45 seconds someone in the United States has a stroke. Each year about 700,000 people experience a new or current stroke ${ }^{5}$. About 500,000 of these are first attack and 200,000 are recurrent attacks ${ }^{4}$. Each year about 46,000 more men than women have a stroke ${ }^{6}$. Men's stroke incidence rate is 1.25 times greater than women's ${ }^{6}$. 


\section{Risk Factors for Stroke}

Risk factors are certain characteristic that give an increase likelihood of having stroke. Stroke is associated with several risk factors, some of them are irreversible and some are modifiable.

\section{Table: Modifiable and Non-modifiable risks factors of} Stroke $^{8}$

\author{
Irreversible risk factors \\ 1. Age \\ 2. Gender $(M>F$ except very young and very old $)$ \\ 3. Race (Afro-Caribbean $>$ Asian $>$ European) \\ 4. Heredity
}

Modifiable risk factors

1. Hypertension

2. Heart disease

a. Heart failure

b. mitral valvular diseases

c. acute myocardial infarction

d. atrial fibrillation

3. Diabetes mellitus

4. Hyperlipidaemia

5. Obesity

6. Smoking

7. Excess alcohol consumption

8. Polycythaemia

9. Oral contraceptive

\section{Irreversible or Non-modifiable Risk Factors}

Age: The incidence of stroke increases with age and approximately doubles with each decade between ages 45 to 85 years. It is uncommon below the age of 40 years ${ }^{9}$. The risk of stroke is greatest in the age group 55-64 years. In UK, stroke causes approximately 10 deaths per 100000 populations at the age of 40 years but 1000 death per 100000 populations at the age of 75 years 7 . A study on risk factors $^{9}$ showed that age has been identified as markers of risk for stroke, cannot be modified. Age is the single most important risk factor for stroke. For each successive 10 years after age 55, the stroke rate more than doubles in both men and women ${ }^{9}$. A study on the risk of subarachnoid and intracerebral hemorrhages was conducted ${ }^{10}$ in Blacks as compared with Whites and described that young and middle-aged Blacks have a substantially higher risk of subarachnoid or intracerebral hemorrhage than Whites of similar age. These types of stroke are important causes of excess mortality among young and middle-aged Blacks ${ }^{10}$. Yousef et $\mathrm{al}^{11}$ described age is an independent risk factor for the development of intracranial atherosclerosis. The prevalence of intracranial atherosclerosis was demonstrated to increase with each decade of age ${ }^{11}$. It was found in $23 \%$ of those $50-59$ years of age, $43 \%$ of those $60-69$ years of age, $65 \%$ of those $70-79$ years of age and $80 \%$ of those $>80$ years of age ${ }^{10}$.

Gender: There is much less difference in the incidence of stroke between male and female, stroke occurs more often in male than in female. A study ${ }^{9}$ showed, gender as a marker of risk for stroke and cannot be modified. Stroke incidence rates are 1.25 times greater in men, but because women tend to live longer than men, more women than men die of stroke each year ${ }^{9}$. A hospital based study ${ }^{8}$ was conducted and showed that male were slightly predominant than female (51\% vs. $49 \%$ ) with age ranging from $21-78$ years and mean age was 50 years. Female were rather older with mean age of 53 years with ranging from 24 to 83 years $^{8}$. A study11 showed, intracranial stenosis is more common among men, particularly in younger age groups and in particular locations, such as the basilar artery. Cross-sectional population-based and autopsy-based epidemiological studies have observed an increased odds ratio of men for developing the disease.

Race: A study was performed on 'The Strong Heart Study'5 to determine the incidence of stroke and to elucidate stroke risk factors among American Indians and suggested that American-Indians have a higher incidence of stroke ${ }^{14}$. A study was conducted to see the risk of subarachnoid and intracerebral haemorrhages in Blacks as compared with Whites and described that lacks had 2.1 times the risk of subarachnoid haemorrhage of Whites (95\% CI, 1.3 to 3.6) and 1.4 times the risk of intracerebral haemorrhage $(95 \%$ CI 0.9 to 2.1). In those under the age of 75, the risk of intracerebral haemorrhage among Blacks was 2.3 times that of Whites (95\% CI 1.5 to 3.6), whereas the risk among Blacks 75 or older was one fourth that of Whites $(95 \%$ CI 0.1 to 0.8 ). Deaths within 30 days of intracerebral or subarachnoid haemorrhage accounted for 1.9 years of life lost per 1000 Blacks under 65 years of age, as compared with 0.5 year per 1000 Whites. Young and middle-aged Blacks had a substantially higher risk of subarachnoid or intracerebral haemorrhage than Whites of similar age. These types of stroke are important causes of excess mortality among young and middle-aged Blacks ${ }^{10}$. A study $^{11}$ demonstrated that, intracranial stenosis is more common in African and Hispanic Americans compared to White Americans. The incidence is even much higher in the Asian population. For example, the relative risk of having intracranial stenosis was 5-fold greater in American Blacks and 5.85-fold greater in Hispanics compared to American Whites. This is supported by data from the Northern Manhattan Stroke Study that showed the likelihood for American Blacks and Hispanics to develop ischaemic stroke was nearly 8-fold higher than Whites. This racial difference in the prevalence of intracranial stenosis was also demonstrated in autopsy studies that found African-Americans had intracranial atherosclerosis more frequently compared to Whites. Whites had more atherosclerosis in the aorta and coronary arteries. Another autopsy study showed African-Americans had more frequent disease at the origin of the ICA. Furthermore, the Asian population is at increased risk for developing intracranial stenosis compared to subjects of Northern European descent. In fact, it is also the most common cerebrovascular lesion in the Asian population. 
Heredity: A study on risk factors, ethnicity and heredity has been identified as markers of risk for stroke. Although these factors cannot be modified, their presence help identify those at greatest risk ${ }^{9}$. A study ${ }^{11}$ showed, multiple genetic factors have been identified that may enhance and promote premature atherosclerosis in the whole vascular system including intracranial large arteries. These include angiotensin converting enzyme polymorphism, plasma to vascular endothelial growth factor ratio, glutathione Stransferase, omega-1 gene polymorphism and plasma homocysteine level. They exert their action via various mechanisms such as vascular endothelial injury, proliferation of vascular smooth muscle cell and impairment of angiogenesis.

\section{Modifiable risk factors}

A hospital based study described risk factors for cerebral infarction in terms of hypertension, diabetes mellitus, ischaemic heart disease, smoking, dyslipidaemia, transient ischemic attack (TIAs), carotid artery stenosis and family history of stroke. They stated that more than $80 \%$ had more than one risk factors, the commonest risk factor hypertension was $55 \%$, positive history of smoking was $30 \%{ }^{5}$. Ischaemic heart disease was found in $34 \%$, diabetes mellitus in $26 \%$, dyslipidaemia in $30 \%$, atrial fibrillation in $25 \%$, carotid artery stenosis in $27 \%^{11} .15 \%$ patients were obese and family history of stroke was $12 \%{ }^{12}$. They concluded that cerebral infarction accounts for 80 to $85 \%$ cases of stroke ${ }^{8}$.

Hypertension: Hypertension is the single most important modifiable risk factor for ischaemic stroke. Most estimates for hypertension indicate a relative risk of stroke of approximately 4 when hypertension is defined as systolic blood pressure $160 \mathrm{~mm} \mathrm{Hg}$ and/or diastolic blood pressure $95 \mathrm{~mm} \mathrm{Hg}$. A summary of seven studies assigning a relative risk of 1 for borderline or mild hypertension determined the relative risk to be about 0.5 at a blood pressure of $136 / 84 \mathrm{~mm} \mathrm{Hg}$ and about 0.35 at a blood pressure of 123/76 $\mathrm{mm} \mathrm{Hg}$. From the lowest to the highest level of blood pressure in this summary, risk is increased about 10-fold. Although clearly important even in the elderly, the impact of hypertension may decrease with age: the odds ratio is 4 at age 50, decreasing to 1 by age 90 . From population surveys the prevalence of hypertension is about $20 \%$ at age 50 , about $30 \%$ at age $60,40 \%$ at age 70 , $55 \%$ at age 80 and $60 \%$ at age 90 . When the Joint National Committee V definition is used $(140 / 90 \mathrm{~mm} \mathrm{Hg}$ or on antihypertensive medication), prevalence increases to about $45 \%$ at age $50,>60 \%$ at age 60 , and $>70 \%$ at age 70 . The prevalence of hypertension is greater in Blacks than in Whites. The efficacy of antihypertensive treatment has been well established in clinical trials. In a summary of 17 treatment trials of hypertension throughout the world involving nearly 50,000 patients, there was a $38 \%$ reduction in all strokes and a $40 \%$ reduction in fatal stroke favoring systematic treatment of hypertension. This effect was true in Whites and Blacks and at all ages. Treatment was also highly effective in preventing stroke in elderly persons with isolated systolic hypertension (Systolic Hypertension in the Elderly Program [SHEP]), the most prevalent form of hypertension in persons older than 65 . Importantly, there was no less impact on stroke prevention above age 80 , with incidence reduced by $40 \%{ }^{9}$. A study ${ }^{11}$ showed that hypertension is the major independent modifiable risk factor for intracranial stenosis. In fact, the impact of hypertension as a risk factor for intracranial atherosclerosis was already established from autopsy studies. Based on epidemiological studies, hypertension is associated with increased odds for the development of intracranial stenosis that ranged from 5 to $9.7^{12}$. Moreover, the risk further increased when hypertension was associated with other risk factors.

Heart disease: Various cardiac diseases have been shown to increase risk of stroke. Atrial fibrillation (AF) is the most powerful and treatable cardiac precursor of stroke. The incidence and prevalence of AF increase with age ${ }^{13}$. With each successive decade of life above age 55, incidence of $\mathrm{AF}$ doubles ${ }^{14}$. Using data from four population-based studies and the US census, it has been estimated that 2.2 million Americans have intermittent or sustained $\mathrm{AF}^{14}$. Prevalence above age 65 is estimated to be $5.9 \%{ }^{15}$. Data from the Framingham Study and hospital discharges suggest that the prevalence of AF in the US population is increasing. The aging of the US population, the increasing incidence of AF with age and the increasing prevalence of AF suggest that AF will result in increasing rates of morbidity and mortality in the population ${ }^{9}$. It is estimated that almost half of all cardioembolic strokes occur in the setting of AF. In the Framingham Study, nonvalvular AF was independently associated with a threefold to fivefold increased risk for stroke ${ }^{17}$. The impact of hypertension, coronary heart disease, and cardiac failure on risk of stroke declined with advancing age, while the impact of AF persisted even into the ninth decade of life. The attributable risk of AF for stroke rose from $1.5 \%$ in subjects aged 50 to 59 years to $23.5 \%$ in subjects aged 80 to 89 years i.e. nearly one stroke in four in persons older than 80 was a result of $\mathrm{AF}^{18}$.

Diabetes mellitus: Persons with diabetes mellitus have an increased susceptibility to atherosclerosis and an increased prevalence of atherogenic risk factors, notably hypertension, obesity and abnormal blood lipids ${ }^{19}$. Casecontrol studies of stroke patients and prospective epidemiological studies have confirmed an independent effect of diabetes mellitus with a relative risk of ischaemic stroke in persons with diabetes mellitus from 1.8 to 3.09 . Among Hawaiian Japanese men in the Honolulu Heart Program, those with diabetes mellitus had twice the risk of thromboembolic stroke of persons without diabetes mellitus that was independent of other risk factors. In a population-based cohort in Rancho Bernardo, persons with diabetes mellitus had a risk-factor adjusted relative risk of stroke of 1.8 in men and 2.2 in women ${ }^{20}$. In Framingham, persons with glucose intolerance have double the risk of brain infarction of nondiabetic persons ${ }^{21}$. In addition to the 
role of glucose status (normal, impaired glucose tolerance or diabetic), there are other aspects of glucose metabolism that may play a role as a risk factor for ischaemic stroke specifically hyperinsulinaemia and increased insulin resistance the relative inability of insulin to enhance glucose disposal ${ }^{22}$. Both were shown to be risk factors for ischaemic stroke among subjects with normal glucose status. In non-Hispanic White and Hispanic subjects, increased insulin resistance is associated with increased atherosclerosis of the carotid arteries independent of glucose status, insulin levels and other major cardiovascular risk factors ${ }^{23}$. A study ${ }^{24}$ showed, diabetes mellitus is an independent risk factor for intracranial stenosis. It promotes the accelerated formation of atherosclerotic stenosis through a decrease in fibrinolytic activity. Based on epidemiological studies, the odds ratio associated with diabetes mellitus ranges from 4 to $5.9^{20}$. In fact, data from the Northern Manhattan Stroke study revealed that patients with intracranial atherosclerosis had a higher prevalence of diabetes mellitus (67\%) when compared to those with extracranial atherosclerosis nonatherosclerotic ( $60 \%$ and $48 \%$ respectively) $)^{26}$. Additionally, the impact of diabetes was also established in autopsy study done in Hong Kong ${ }^{27}$. Based on these studies, it may be the strongest risk factor for intracranial stenosis.

Hyperlipidaemia: Hypercholesterolaemia is an important modifiable risk factor for coronary heart disease, the link to ischaemic stroke remains uncertain. Data clearly support the positive relation between total and LDL cholesterol and a protective influence of HDL cholesterol on extracranial carotid atherosclerosis ${ }^{19}$. Lipids give some contribution to stroke risk even in the elderly, with a more prevalent role for HDL than LDL and that lipid profile assessment must be taken into account in estimating the individual risk of stroke $^{20}$. Strong correlation between plasma lipoproteins concentration and risk of stroke has never been clearly established. Despite the incontrovertible relationship between elevated serum cholesterol and lipids and coronary artery disease, no conclusive evidence links lipid abnormalities to stroke, nevertheless most authorities strongly advice stroke prone patients to lower the elevated blood cholesterol and triglyceride level. Low levels of high-density lipoprotein cholesterol $(<0.90 \mathrm{mmol} / \mathrm{L})$, high levels of total triglyceride $(>2.30 \mathrm{mmol} / \mathrm{L})$ and the presence of hypertension were associated with a twofold increase in the risk of stroke mortality or morbidity ${ }^{21}$. A study ${ }^{11}$ showed, intracranial stenosis has been associated with dyslipidemia, specifically elevated total cholesterol but also its various components. High lipoprotein is an independent marker for a greater extent of disease. There is actually a synergic effect between lipoprotein "a" and diabetes mellitus and resultant intracranial occlusive disease. Elevated LDL has also been shown to be a risk factor for intracranial stenosis.

Obesity: Obesity is clearly related to hypertension and an increased weight is often associated with increase in severity of hypertension. A study in Japan shows that obesity is positively correlated with stroke incidence ${ }^{15}$. Excess bodyweight is the sixth most important risk factor contributing to the overall burden of disease worldwide. 1.1 billion adults and $10 \%$ of children are now classified as overweight or obese. Average life expectancy is already diminished; the main adverse consequences are cardiovascular disease, type 2 diabetes and several cancers. Obesity, with its array of comorbidities, necessitates careful clinical assessment to identify underlying factors and to allow coherent management ${ }^{22}$.

Smoking: Cigarette smoking increases risk (RR) of ischaemic stroke nearly two times with a clear doseresponse relation. In both the Framingham Study and the Nurses' Health Study cessation of smoking led to a prompt reduction in stroke risk-major risk was reduced within 2 to 4 years. This reduction in risk occurred throughout the age spans of these studies and in heavy as well as moderate smokers $^{23}$.

Alcohol consumption: Ischaemic stroke showed a curvilinear relationship with a protective effect of alcohol for low to moderate consumption and increased risk for higher exposure. For more than 3 drinks on average/day, in general women had higher risks than men and the risks for mortality were higher compared to the risks for morbidity. Heavy alcohol consumption increases the relative risk of any stroke while light or moderate alcohol consumption may be protective against ischaemic stroke ${ }^{24}$. Increased risk for small-artery occlusion in ischaemic stroke was associated with frequent alcohol intake ${ }^{25}$.

Oral contraceptive: Oral contraceptives with an estrogen content $>50 \mu \mathrm{g}$, the preparations used in the 1960s and 1970 s, were strongly associated with risk for stroke. Recently a study of low-dose oral contraceptives $(<50 \mu \mathrm{g}$ estrogen) disclosed no increased risk of stroke in more than 3.6 million woman-years of observation ${ }^{26}$.

\section{Conclusion}

There are several modifiable and non-modifiable risk factors of stroke which are preventable.

\section{References}

1. Hatano S. Variability of the diagnosis of stroke by clinical judgment and by a scoring method. Bulletin of the World Health Organization 1976;54(5):533.

2. Schellinger PD, Kaste M, Hacke W. An update on thrombolytic therapy for acute stroke. Current opinion in neurology 2004;17(1):69-77

3. Wahlgren N, Ahmed N, DÃ $j$ valos A, Hacke W, MillÃ $; n$ Mn, Muir K, et al. Thrombolysis with alteplase 3 4-5 h after acute ischaemic stroke (SITSISTR): an observational study. The Lancet 2008;372(9646):1303-1309 4. Bamford JM, Sandercock PAG, Dennis MS, Burn JPS, Warlow CP. A prospective study of acute cerebrovascular disease in the community: the Oxfordshire Community Stroke Project--1981-86. 2. Incidence, case fatality rates and overall outcome at one year of cerebral infarction, primary intracerebral and subarachnoid haemorrhage. Journal of Neurology, Neurosurgery \& Psychiatry 1990;53(1):16-22

5. McNaughton H, Weatherall M, Taylor W, McPherson K. Factors influencing rate of Barthel Index change in hospital following stroke. Clinical rehabilitation 2001;15(4):422-427

6. Collis T, Devereux RB, Roman MJ, de Simone G, Yeh J-L, Howard 
BV, et al. Relations of Stroke Volume and Cardiac Output to Body Composition The Strong Heart Study. Circulation 2001;103(6):820-825.

7. White, Halina, et al. "Ischemic stroke subtype incidence among whites, blacks, and hispanics the northern manhattan study." Circulation 2005;111(10): 1327-1331.

8. Chambless, Lloyd E., et al. "Carotid wall thickness is predictive of incident clinical stroke: the Atherosclerosis Risk in Communities (ARIC) study." American Journal of Epidemiology 2000;151(5): 478-487.

9. Miah, M. N. A., M. A. Azhar, A. Rahman, D. Halder, M. Akteruzzaman, and N. C. Kundu. "Risk Factors of Stroke in Young and Old age Group-A Comparative Study." Journal of Medicine 2012;13(2): 138-142.

10. Sacco RL, Kargman DE, Gu Q, Zamanillo MC. Race-ethnicity and determinants of intracranial atherosclerotic cerebral infarction The Northern Manhattan Stroke Study. Stroke 1995;26(1):14-20.

11. Broderick, Joseph P., et al. "Relationship of cardiac disease to stroke occurrence, recurrence, and mortality." Stroke (1992) 23.9: 1250-1256.

12. Mohammad, Yousef, Marwan Qattan, and Shyam Prabhakaran. "Epidemiology and pathophysiology of intracranial large artery stenosis." stroke 2010; 3: 4.

13. Solberg, L. A., and P. A. McGarry. "Cerebral atherosclerosis in Negroes and Caucasians." Atherosclerosis 1972;16(2): 141-154.

14. Lee, E. J., et al. "Relevance of common carotid intima-media thickness and carotid plaque as risk factors for ischemic stroke in patients with type 2 diabetes mellitus." American journal of neuroradiology 2007; 28(5): 916-919. 15. Yang, Yi, and Gary A. Rosenberg. "Blood-brain barrier breakdown in acute and chronic cerebrovascular disease." Stroke 2011; 42(11): 3323-3328. 16. Zhang, Wanqing, and Shinobu Watanabe?Galloway. "Ten?Year Secular Trends for Congestive Heart Failure Hospitalizations: An Analysis of Regional Differences in the United States." Congestive Heart Failure 2008; 14(5): 266-271.

17. Shinozaki, Kazuya, et al. "Role of insulin resistance associated with compensatory hyperinsulinemia in ischemic stroke." Stroke 1996;27(1): 37-43. 18. Rincon, Fred, et al. "Incidence and risk factors of intracranial atherosclerotic stroke: the Northern Manhattan Stroke Study." Cerebrovascular diseases (Basel, Switzerland) 2009; 28(1): 65.

19. Baker, Andrew J., et al. "Excitatory amino acids in cerebrospinal fluid following traumatic brain injury in humans." Journal of neurosurgery 1993; 79(3): 369-372.

20. Iso, Hiroyasu, et al. "Serum cholesterol levels and six-year mortality from stroke in 350,977 men screened for the multiple risk factor intervention trial." New England Journal of Medicine 1989; 320(14): 904-910.

21. Denti, Licia, et al. "The role of lipid profile in determining the risk of ischemic stroke in the elderly: a case-control study." Archives of gerontology and geriatrics 2003; 37(1): 51-62.

22. Seppo, Leena, et al. "A fermented milk high in bioactive peptides has a blood pressure-lowering effect in hypertensive subjects." The American journal of clinical nutrition 2003; 77(2): 326-330.

23. Jetten, Jolanda, Catherine Haslam, Cara Pugliese, James Tonks, and S. Alexander Haslam. "Declining autobiographical memory and the loss of identity: Effects on well-being." Journal of Clinical and Experimental Neuropsychology 2010; 32(4): 408-416.

24. Shinton, Roger, and Gareth Beevers. "Meta-analysis of relation between cigarette smoking and stroke." Bmj 1989; 298(6676): 789-794.

25. Patra, Jayadeep, et al. "Alcohol consumption and the risk of morbidity and mortality for different stroke types-a systematic review and metaanalysis." BMC Public Health 2010; 10(1): 258.

26. Song, Yun-Mi, et al. "Different risk factor profiles between subtypes of ischemic stroke. A case-control study in Korean men." European journal of epidemiology 2005; 20(7): 605-612.

27. Petitti, Diana B., et al. "Stroke in users of low-dose oral contraceptives." New England journal of medicine 1996;335(1): 8-15 